\title{
Generalized Semi-Analytical Solution of Advection- Diffusion-Reaction in Finite and Semi-Infinite Cylindrical Ducts
}

\author{
Yashar Mehmani*, Matthew T. Balhoff* \\ * Department of Petroleum and Geosystems Engineering, The University of Texas at Austin, 200 \\ E. Dean Keeton St., Stop C0300, Austin, Texas 78712-1585, United States
}

\begin{abstract}
A semi-analytical solution for the transient advection-diffusion-reaction problem within finite and semi-infinite ducts is derived. The solution allows for general radial- and time-dependent inlet/outlet conditions, complex boundary conditions on the duct wall including adsorption and decay, and arbitrary velocity profiles of the transporting fluid. The only numerical step of the solution is the inverse Laplace transform in the time variable. Therefore, the approach also produces fully analytical steady-state solutions. The solution is verified against computational fluid dynamics (CFD) simulations under various boundary conditions and velocity profiles (Newtonian and power-law), and in all cases good agreement is obtained. Although theoretically applicable to all regimes, the solution is computationally difficult at very high Peclet numbers and very early times due to numerical instabilities as a result of finite precision arithmetic of computers. A convergence analysis is conducted to delineate the boundaries of this limit for two important cases.

The solution was derived using a new approach for solving two-dimensional partial differential equations (PDEs) with non-constant coefficients which parallels the Frobenius and power series methods for solving ordinary differential equations (ODEs). The approach reduces the original PDE to a single infinite-order ODE with constant coefficients. The approach is suspected to provide solutions to a large class of PDEs of this type. The solution may find applications in a number of engineering and/or biomedical fields, it can be used to verify numerical simulators, and serve as a simple and easy-toimplement alternative where access to numerical simulators is not available.
\end{abstract}

Keywords. heat and mass transfer, solute transport, cylindrical duct, analytical solution, partial differential equations, Frobenius method

\section{Introduction}

The problem of heat and mass transfer within cylindrical tubes has been examined for over a century since Graetz considered a simplified version of the problem in 1883 (Graetz, 1883). His work and those that have followed since consider the steady-state, fully developed temperature profile of a fluid flowing in a constant-wall-temperature duct. The problem has relevance in the design and analysis of heat exchangers. Graetz had originally considered the steady-state 
problem ignoring axial conduction (or diffusion). An analytical solution including this term was recently developed by Lahjomri and Oubarra (1999). Their method employs an approach similar to the method of separation of variables, with the difference that the Eigen-functions do not form an orthogonal set. The elegance of their approach is that despite the non-orthogonally of the Eigen-bases the "Fourier-coefficients" are explicitly recovered. The solution has been further extended to scenarios with added physics, such as viscous dissipation and slip flow (e.g. Barron et al., 1997; Jeong and Jeong, 2006) using very similar approaches.

The problem of solute dispersion within cylindrical ducts appears to be first considered by Taylor (1953), who showed that the late-time dispersal of solutes follows Fickian behavior. Aris (1956) later extended Taylor's theory by including axial diffusion into his analysis (originally ignored by Taylor). The dispersion problem was later extensively studied by various investigators, both with the aim of extending Taylors theory and delineating its limits e.g. Ananthakrishnan et al. (1965), Gill and Sankarasubramanian (1970). The majority of the work on dispersion concerns itself with the cross-sectional average of the concentration. However, there are scenarios (e.g. ascertaining the local Sherwood number along the duct wall) where knowledge of the local concentration field becomes important. The solution of the unsteady-state transport equation for the local concentration field has been attempted by a handful of investigators. Lighthill (1966) derived a simple and elegant analytical solution applicable to early times when the stretching solute has not had enough time to sense the walls of the duct. The applicability of his solution becomes important at large Peclet numbers. Chun (1970) and Hunt (1977) both used perturbation analysis to obtain solutions limited to very low $(P e<0.1)$ and very high Peclet numbers, respectively. Tseng and Besant (1972) derived an exact solution for the solute transport equation in an infinite tube with impulse and step-change initial conditions. The solution involved an expansion with respect to zero-order Bessel functions (of first kind) in the radial component which transformed the differential equation into a matrix form. In the process, Eigenvalues and Eigenvectors of the corresponding matrix had to be computed and, overall, the solution was rather numerically involved.

Perhaps the most comprehensive solution and analysis done so far was by $\mathrm{Yu}$ in a series of papers $(1976,1979$, and 1981). His approach was to similarly expand the solution in terms of zero-order Bessel functions (of first kind) in the radial component. This converted the original differential equation into an infinite system of coupled second order differential equations in infinite unknowns. However, for an infinite tube $\mathrm{Yu}$ was able to use the Fourier and the Laplace transforms to obtain solutions for impulse and step change initial conditions. The resulting solution was numerically involved and required quite a bit of computational effort. Shankar and Lenhoff (1989) remedied this problem by replacing the Fourier transform step of Yu by a Fourier expansion. However, they had to ignore the axial diffusion term in the process. That said $\mathrm{Yu}$ showed his approach could reproduce the Taylor limit, provided ample comparison with almost all previous models developed in the literature, and showed his solution was equivalent to that of Tseng and Besant (1972). Although his analysis showing the equivalence of his approach to that 
of Gill and Sankarasubramanian (1970) was later disputed by Gill and Sankarasubramanian (1980) and shown to be incorrect.

All the literature mentioned above, provide solutions for the case of an infinite tube with no boundary conditions imposed in the axial direction (other than decay conditions at infinity). In fact, the authors of this work are unaware of any work that provides analytical treatments of finite length pipes with prescribed axial inlet/outlet conditions. This is understandable since analysis of the problem becomes even more difficult for finite length ducts and previous approaches, for the most part, become inapplicable. Furthermore, previous work is limited to very specific and rather simple boundary conditions imposed on the duct wall. The goal of the current work is to present a new semi-analytical solution that has the flexibility to be applied to a vast majority of heat/mass transfer problems in cylindrical ducts (as well as Cartesian slits). It is applicable to both finite and semi-infinite tubes and allows for general boundary conditions at the duct wall, inlet and outlet. The solution is semi-analytical because it involves a numerical Laplace inverse transform step in the time variable (which is numerically simple). For steadystate scenarios our approach produces fully-analytical solutions due to the absence of the Laplace transform step. The solution approach appears to be applicable to a wide class of partial differential equations (PDEs) (in both Cartesian and cylindrical coordinates) with variable coefficients, and parallels (and seems to extend) the Frobenius and power series methods for solving ordinary differential equations (ODEs).

\section{Problem Statement}

The transport of a dilute solute species under laminar flow of a single-phase Newtonian fluid within a cylindrical duct and subject to first-order decay is described by eq. 1. We assume negligible entrance effects and assume fully developed flow starting from the pipe inlet, which is a good assumption for sufficiently long pipes and sufficiently small Reynolds numbers (exact quantification of the entrance length can be found in Durst et al., 2005). Additional assumptions include isothermal flow and a concentration-independent molecular diffusion coefficient. If the species is allowed to adsorb onto the walls of the duct and undergo an independent decay process, eq. 2 is additionally needed to describe the rate of change of the adsorbed solute. Note that the form of eq. 1 remains unchanged in the description of heat transfer under similar assumptions, in which case concentration needs to be replaced by temperature. In this case, eq. 2 , however, requires an additional diffusion term arising from the conduction of heat along the duct wall. This term will not be included in the analysis of this paper (since the focus is primarily on solute transport) and requires further analytic treatment. The solution approach presented in section 3 is not limited to parabolic fluid velocity profiles (as it appears in eq. 1). However, to keep the analysis simple we demonstrate the approach for a Newtonian fluid. An example of a non-Newtonian (power-law) fluid is discussed in section 4.4. 
$\frac{\partial \tilde{c}}{\partial t}+V_{0}\left(1-\left(\frac{r}{R}\right)^{2}\right) \frac{\partial \tilde{c}}{\partial x}=D_{m}\left(\frac{1}{r} \frac{\partial}{\partial r}\left(r \frac{\partial \tilde{c}}{\partial r}\right)+\frac{\partial^{2} \tilde{c}}{\partial x^{2}}\right)-\omega \tilde{c}$

$\delta \frac{\partial \tilde{\phi}}{\partial t}=-\left.D_{m} \frac{\partial \tilde{c}}{\partial r}\right|_{r=R}-\gamma \phi \delta$

In eq. 1 and 2, $\tilde{c}$ is the concentration within the fluid bulk, $\tilde{\phi}$ is the concentration of the adsorbed solute onto the duct wall, $V_{0}$ is the maximum centerline fluid velocity, $D_{m}$ is the molecular diffusion coefficient, $\omega$ is the rate of decay within the fluid bulk, $\delta$ is the pipe wall thickness, $\gamma$ the rate of decay of the adsorbed solute, and $R$ is the duct radius. Equations 1 and 2 are subject to the following initial and general boundary conditions:

$$
\begin{aligned}
& \tilde{c}(t=0, x, r)=0 \quad \tilde{\phi}(t=0, x)=0 \\
& \left.\frac{\partial \tilde{c}}{\partial r}\right|_{r=0}=0 \\
& \left.-\left.D_{m} \frac{\partial \tilde{c}}{\partial r}\right|_{r=R}=h\left(\left.\tilde{c}\right|_{r=R}-\tilde{\phi}\right) \quad \text { (special cases: }\left.\frac{\partial \tilde{c}}{\partial r}\right|_{r=R}=0 \text { and }\left.\tilde{c}\right|_{r=R}=0\right) \\
& \left.\tilde{a}_{1} \frac{\partial \tilde{c}}{\partial x}\right|_{x=0}+\left.\tilde{a}_{2} \tilde{c}\right|_{x=0}=f(t) \\
& \begin{cases}\tilde{b} & \left.\frac{\partial \tilde{c}}{\partial x}\right|_{x=L}+\left.\tilde{b_{2}} \tilde{c}\right|_{x=L}=g(t) \quad \text { if } x \in[0, L] \\
\tilde{c}, \frac{\partial \tilde{c}}{\partial x} \rightarrow 0 \quad \text { as } \quad x \rightarrow \infty & \text { if } x \in[0, \infty)\end{cases}
\end{aligned}
$$

Eq. $3 \mathrm{~b}$ expresses the axial symmetry of the concentration profile across the duct cross-section. Eq. $3 \mathrm{c}$ relates the rate of mass transfer at the duct wall to the concentration difference between the fluid bulk and the adsorbed solute ( $h$ is the mass transfer coefficient). To allow analytic treatment, this boundary condition assumes linear first-order adsorption rates. Two special cases of this boundary condition are also given within parentheses in eq. 3c. Eq. $3 \mathrm{c}$ is responsible for the coupling of eq. 1 and 2. Eq. $3 \mathrm{~d}$ and $3 \mathrm{e}$ describe general boundary conditions imposed at the inlet and outlet of a finite pipe. $a_{1}, a_{2}, \tilde{b}_{1}$ and $\tilde{b}_{2}$ can be, in their most general form, functions of $r$ (the radial coordinate) (typically related to physical attributes of the system) without setting any limitations on the analysis provided in section 3. For convenience, they are assumed to be constant parameters throughout the paper unless stated otherwise. A discussion pertaining to cases where these parameters are dependent on $r$ is provided at the end of section 3. For similar reasons, $f$ and $g$ are assumed to be arbitrary functions of time alone, whereas they can generally depend on $r$ as well. For a semi-infinite pipe the outlet condition is replaced by the 
limiting condition given in eq. 3e. In order to reduce the number of free parameters and present our final results in a more useful format, eq. 1-3 are non-dimensionalized with the following choice of non-dimensional variables.

$$
\begin{aligned}
& \xi=\frac{r}{R} \quad \lambda=\frac{x}{L} \quad \tau=\frac{t V_{0}}{L} \quad c=\frac{\tilde{c}}{\tilde{c}_{0}} \quad \phi=\frac{\tilde{\phi}}{\tilde{c}_{0}} \quad \kappa=\left(\frac{L}{R}\right)^{2} \quad Z=\frac{D_{m} L}{V_{0} R \delta} \\
& P e=\frac{V_{0} L}{D_{m}} \quad S h=\frac{R h}{D_{m}} \quad D a_{I}=\frac{L \omega}{V_{0}} \quad D a_{I I}=\frac{\gamma L}{V_{0}}
\end{aligned}
$$

The characteristic concentration $\tilde{c}_{0}$ is typically chosen as the maximum concentration value attainable within the system. In the results presented in section 4 , it is chosen as the (constant) inlet concentration value. The resulting dimensionless differential equations are eq. 5 and 6 :

$$
\begin{aligned}
& \frac{\partial c}{\partial \tau}+\left(1-\xi^{2}\right) \frac{\partial c}{\partial \lambda}=\frac{\kappa}{P e} \frac{1}{\xi} \frac{\partial}{\partial \xi}\left(\xi \frac{\partial c}{\partial \xi}\right)+\frac{1}{P e} \frac{\partial^{2} c}{\partial \lambda^{2}}-D a_{I} c \\
& \frac{\partial \phi}{\partial \tau}=-\left.Z \frac{\partial c}{\partial \xi}\right|_{\xi=1}-D a_{I I} \phi
\end{aligned}
$$

Subject to the following dimensionless initial and boundary conditions:

$$
\begin{aligned}
& c(\tau=0, \lambda, \xi)=0 \quad \phi(\tau=0, \lambda)=0 \\
& \left.\frac{\partial c}{\partial \xi}\right|_{\xi=0}=0 \\
& \left.\frac{\partial c}{\partial \xi}\right|_{\xi=1}=-S h\left(\left.c\right|_{\xi=1}-\phi\right) \quad\left(\text { special cases: }\left.\frac{\partial c}{\partial \xi}\right|_{\xi=1}=0 \quad \text { and }\left.\quad c\right|_{\xi=1}=0\right) \\
& \left.a_{1} \frac{\partial c}{\partial \lambda}\right|_{\lambda=0}+\left.a_{2} c\right|_{\lambda=0}=f(\tau) \\
& \begin{cases}\left.b_{1} \frac{\partial c}{\partial \lambda}\right|_{\lambda=1}+\left.b_{2} c\right|_{\lambda=1}=g(\tau) \quad \text { if } \lambda \in[0,1] \\
c, \frac{\partial c}{\partial \lambda} \rightarrow 0 \quad \text { as } \quad \lambda \rightarrow \infty \quad \text { if } \lambda \in[0, \infty)\end{cases}
\end{aligned}
$$

In eq. 5-7, $S h$ is the Sherwood (or Nusselt in heat transfer) number (mass transfer over diffusion), $P e$ is the Peclet number (advection over diffusion), and $\kappa$ is the aspect ratio of the finite duct. $D a_{I}$ and $D a_{I I}$ denote Damköhler numbers (reaction over advection) associated with the fluid bulk and the pipe wall respectively. It should be noted that for semi-infinite ducts $L$ is replaced by $R$ in all 
the equations above (eq. 4-7) without any impact on the following mathematical analysis. Other parameters arising within eq. 5-7 are defined in eq. 7f. The functions $f$ and $g$ are simply obtained by transforming the time variable $t$ into $\tau$ within $f$ and $g$.

$$
a_{1}=\frac{a_{1} \tilde{c}_{0}}{L} \quad a_{2}=a_{2} \tilde{c}_{0} \quad b_{1}=\frac{\tilde{b}_{1} \tilde{c}_{0}}{L} \quad b_{2}=\tilde{b}_{2} \tilde{c}_{0}
$$

\section{Problem Solution}

As the first step the Laplace transforms of $c$ and $\phi$ is taken with respect to the time variable (i.e.

$\tau)$. The Laplace transform is defined as:

$$
\hat{c}(T, \lambda, \xi)=\int_{0}^{\infty} c(\tau, \lambda, \xi) e^{-T \tau} d \tau \quad \phi(T, \lambda)=\int_{0}^{\infty} \phi(\tau, \lambda) e^{-T \tau} d \tau
$$

Thus eq. 5, 6 and 7 respectively become:

$$
\begin{aligned}
& \left(T+D a_{I}\right) \hat{c}+\left(1-\xi^{2}\right) \frac{\partial \hat{c}}{\partial \lambda}=\frac{\kappa}{P e} \frac{1}{\xi} \frac{\partial}{\partial \xi}\left(\xi \frac{\partial \hat{c}}{\partial \xi}\right)+\frac{1}{P e} \frac{\partial^{2} \hat{c}}{\partial \lambda^{2}} \\
& \phi=-\left.\frac{Z}{\left(D a_{I I}+T\right)} \frac{\partial \hat{c}}{\partial \xi}\right|_{\xi=1} \\
& \left.\frac{\partial \hat{c}}{\partial \xi}\right|_{\xi=0}=0 \\
& \phi=\left.\frac{1}{S h} \frac{\partial \hat{c}}{\partial \xi}\right|_{\xi=1}+\left.\hat{c}\right|_{\xi=1} \\
& \left.a_{1} \frac{\partial \hat{c}}{\partial \lambda}\right|_{\lambda=0}+\left.a_{2} \hat{c}\right|_{\lambda=0}=F(T) \\
& \begin{cases}\left.b_{1} \frac{\partial \hat{c}}{\partial \lambda}\right|_{\lambda=1}+\left.b_{2} \hat{c}\right|_{\lambda=1}=G(T) & \text { if } \lambda \in[0,1] \\
\hat{c}, \frac{\partial \hat{c}}{\partial \lambda} \rightarrow 0 \quad \text { as } \lambda \rightarrow \infty & \text { if } \lambda \in[0, \infty)\end{cases}
\end{aligned}
$$

Combining eq. 10 and $11 \mathrm{~b}$ we obtain: 
$\left.\left(\frac{1}{S h}+\frac{Z}{D a_{I I}+T}\right) \frac{\partial \hat{c}}{\partial \xi}\right|_{\xi=1}+\left.\hat{c}\right|_{\xi=1}=0$

Thus we readily observe that by taking the Laplace transform we have decoupled eq. 5 from 6 . Now, we formally introduce eq. 13 as the solution to eq. 9 subject to eq. $11 \mathrm{a}, 12,11 \mathrm{c}$ and $11 \mathrm{~d}$. Note that the solution approach that follows bears similarities to the Frobenius method for solving ODEs with variable coefficients. Therefore, the following solution approach may be regarded as an extension to the Frobenius method (or power series method in Cartesian problems) for solving PDEs with variable coefficients, and is expected to be applicable to a large class of PDEs in two-variables. To the authors' knowledge, this is the first time such an extension is proposed (further discussion is provided at the end of this section).

$\hat{c}=\sum_{n=0}^{\infty} A_{n}(\lambda) \xi^{n}$

Substituting eq. 13 into eq. 9 and grouping terms of the same $\xi$-power we obtain:

$$
\begin{array}{ll}
A_{0}(\lambda) \neq 0 & \\
A_{2}(\lambda)=\frac{\left(T+D a_{I}\right) A_{0}+A_{0}^{\prime}-(1 / P e) A_{0}^{\prime \prime}}{(4 \kappa / P e)} & \\
A_{2 m}(\lambda)=\frac{\left(T+D a_{I}\right) A_{2 m-2}+A_{2 m-2}^{\prime}-A_{2 m-4}^{\prime}-(1 / P e) A_{2 m-2}^{\prime \prime}}{(2 m)^{2}(\kappa / P e)} & \\
A_{2 m-1}(\lambda)=0 & \forall m \geq 1
\end{array}
$$

The derivatives appearing in eq. 14 are with respect to the variable $\lambda$. Using eq. 14 all $A_{2 m}$ terms can be written as a linear combination of $A_{0}$ and its derivatives (i.e. eq. 15):

$$
A_{2 m}(\lambda)=\sum_{j=0}^{2 m} \alpha_{2 m, j} A_{0}^{(j)}
$$

The $\alpha_{2 m, j}$ coefficients are readily computed recursively from eq. 14 (note that $\alpha_{0,0}=1$ ). On the other hand, inserting eq. 13 into eq. 12 and eq. 11 a respectively yields:

$$
\begin{aligned}
& A_{0}(\lambda)+\sum_{n=1}^{\infty}\left[1+\left(\frac{1}{S h}+\frac{Z}{T+D a_{I I}}\right) n\right] A_{n}(\lambda)=0 \\
& A_{1}(\lambda)=0
\end{aligned}
$$


Eq. 17 is consistent with eq. 14 obtained from the differential equation 9. Note that the two special cases of boundary conditions at the duct wall (pointed out in eq. $7 \mathrm{c}$ ) yield the following equations instead of eq. 16:

$$
\begin{aligned}
& \sum_{n=1}^{\infty} n A_{n}(\lambda)=0 \\
& \sum_{n=0}^{\infty} A_{n}(\lambda)=0
\end{aligned}
$$

Since all $A_{n}$ terms in eq. 16,18 and 19 can be written in terms of $A_{0}$ and its derivatives, they can be considered as "transcendental equations" in the form of infinite-order ordinary differential equations with respect to the single unknown function $A_{0}(\lambda)$. For computational purposes these equations need to be truncated to a finite number of terms; say $N_{R}$ (an even integer in this case). Therefore, truncating eq. 16 (similarly eq. 18 and 19) and substituting eq. 15 for all $A_{n}$ terms with $n \geq 2$, we obtain the following:

$$
\begin{aligned}
& \sum_{k=0}^{N_{R}} c_{k} A_{0}^{(k)}=0 \\
& c_{k}=\sum_{m=\left[\frac{k+1}{2}\right]}^{N_{R} / 2}\left(1+\left(\frac{1}{S h}+\frac{Z}{T+D a_{I I}}\right)(2 m)\right) \alpha_{2 m, k} \\
& c_{k}=\sum_{m=\left[\frac{k+1}{2}\right]}^{N_{R} / 2}(2 m) \alpha_{2 m, k} \\
& c_{k}=\sum_{m=\left[\frac{k+1}{2}\right]}^{N_{R} / 2} \alpha_{2 m, k}
\end{aligned}
$$

Eq. 20a is a linear, homogeneous, finite-order ordinary differential equation with constant coefficients. The $c_{k}$ coefficients given by eq. 20b, 20c and 20d correspond to eq. 16, 18 and 19 respectively. Note that these coefficients may assume different forms depending on the specific problem at hand, but can be easily derived (e.g. non-Newtonian fluids when odd $A_{n}$ terms are non-zero). In general, these coefficients are complex because the Laplace variable $T$ may be complex. However, the numerical Laplace inversion algorithm used in this work (Stehfest, 1970; explained later in this section) requires function evaluations for which the Laplace variable $T$ is real. In this case, the $c_{k}$ coefficients in eq. 20a can also be expected to be real (because all other parameters involved are real) and we proceed further on this basis. We should note, however, that this by no means prevents one from using inverse algorithms that require function evaluations for complex values of $T$. The difference would be in the possible absence of conjugate roots in the characteristic polynomial (i.e. eq. 21) discussed next. 
Eq. 20a has very simple bases for its solution, which can be constructed from the roots of its characteristic polynomial given by eq. 21 .

$\sum_{k=0}^{N_{R}} c_{k} s^{k}=0$

The roots of this polynomial are easily obtained via computing the Eigenvalues of its companion matrix using a computer. Here, this is readily accomplished by calling the "roots" command in MATLAB. Note that this step is no more difficult than computing the roots of transcendental equations involving Bessel functions (for obtaining the Eigenvalues) in the approaches used by previous authors (e.g. Tseng and Besant, 1972). Depending on whether a given root is real or complex, eq. 22 denotes its corresponding basis $b_{i}$.

$b_{i}(\lambda)= \begin{cases}e^{r_{i} \lambda} & r_{i} \in \square \\ e^{v_{i} \lambda} \sin \left(u_{i} \lambda\right) \& e^{v_{i} \lambda} \cos \left(u_{i} \lambda\right) & v_{i} \pm u_{i} i \in \square\end{cases}$

One may perhaps wish to also consider the possibility of repeated roots. However, the occurrence of repeated roots was never observed during numerous calculations conducted by the authors. This renders the analysis and its implementation into computer code much simpler. The general solution of eq. 20a can now be constructed by linearly combining the bases $b_{i}$ given by eq. 23.

$$
A_{0}(\lambda)=\sum_{i=1}^{N_{R}} \beta_{i} b_{i}(\lambda)
$$

In order to obtain the unknown $\beta_{i}$ coefficients, we substitute eq. 13 into eq. $11 \mathrm{c}$ and $11 \mathrm{~d}$ (and group similar $\xi$-powers) from which we get eq. 24 for the inlet:

$$
\left\{\begin{array}{l}
a_{1} A_{0}^{\prime}(0)+a_{2} A_{0}(0)=F(T) \\
a_{1} A_{n}^{\prime}(0)+a_{2} A_{n}(0)=0
\end{array} \quad \forall n \geq 1\right.
$$

And eq. 25 for the outlet of a finite duct:

$$
\left\{\begin{array}{l}
b_{1} A_{0}^{\prime}(1)+b_{2} A_{0}(1)=G(T) \\
b_{1} A_{n}^{\prime}(1)+b_{2} A_{n}(1)=0
\end{array} \quad \forall n \geq 1\right.
$$

Substituting eq. 15 for all $A_{n}$ terms in eq. 24 and 25, we obtain an infinite set of equations describing the boundary conditions of $A_{0}$ at $\lambda=0$ and 1 for a finite-length duct. Choosing from 
this system an equal number of boundary conditions at $\lambda=0$ as at $\lambda=1$, one can compute the $\beta_{i}$ coefficients in eq. 23. This is done by solving a relatively small (depending on the order of truncation, $N_{R}$ ) system of equations with respect to the unknowns $\beta_{i}$. The treatment is even simpler for semi-infinite ducts. Namely, by substituting eq. 13 into the limiting boundary condition of eq. $11 \mathrm{~d}$ at infinity, we obtain:

$A_{0}^{(n)}(\lambda) \rightarrow 0 \quad$ as $\quad \lambda \rightarrow \infty \quad \forall n \geq 0$

Eq. 26 dictates that bases, $b_{i}$, in which the power of the exponential function is a positive number multiplied by $\lambda$ (i.e. $e^{r_{i} \lambda}$ or $e^{v_{i} \lambda}$ where $r_{i}, v_{i}>0$ ), have a corresponding $\beta_{i}$ coefficient that is zero. This reduces the number of the unknown $\beta_{i}$ coefficients to be calculated. A sufficient number of boundary conditions for $A_{0}$ at $\lambda=0$ is then chosen (from eq. 24) to determine the rest of the $\beta_{i}$ coefficients (by again solving a relatively small system of equations). Since in the process of determining the $\beta_{i}$ coefficients it becomes necessary to accurately calculate the derivatives of the bases (i.e. $b_{i}$ ), eq. 27-30 provide simple (and easy-to-implement) means of determining the $k^{\text {th }}$ derivative of these bases.

$$
\begin{aligned}
& \left(e^{r_{i} \lambda}\right)^{(k)}=r_{i}^{n} e^{r_{i} \lambda} \\
& \left(\sin \left(u_{i} \lambda\right)\right)^{(k)}=\left\{\begin{array}{lll}
(-1)^{k / 2} u_{i}^{k} \sin \left(u_{i} \lambda\right) & k=2 l, & \forall l \geq 0 \\
(-1)^{(k-1) / 2} u_{i}^{k} \cos \left(u_{i} \lambda\right) & k=2 l-1, & \forall l \geq 1
\end{array}\right. \\
& \left(\cos \left(u_{i} \lambda\right)\right)^{(k)}=\left\{\begin{array}{lll}
(-1)^{k / 2} u_{i}^{k} \cos \left(u_{i} \lambda\right) & k=2 l, & \forall l \geq 0 \\
(-1)^{(k+1) / 2} u_{i}^{k} \sin \left(u_{i} \lambda\right) & k=2 l-1, & \forall l \geq 1
\end{array}\right. \\
& \left(e^{v_{i} \lambda} \sin \left(u_{i} \lambda\right)\right)^{(k)}=\sum_{j=0}^{k}\left(\begin{array}{c}
k \\
j
\end{array}\right)\left(e^{v_{i} \lambda}\right)^{(k-j)}\left(\sin \left(u_{i} \lambda\right)\right)^{(j)} \\
& \left(e^{v_{i} \lambda} \cos \left(u_{i} \lambda\right)\right)^{(k)}=\sum_{j=0}^{k}\left(\begin{array}{c}
k \\
j
\end{array}\right)\left(e^{v_{i} \lambda}\right)^{(k-j)}\left(\cos \left(u_{i} \lambda\right)\right)^{(j)}
\end{aligned}
$$

Once the $\beta_{i}$ coefficients are calculated $A_{0}$ is automatically obtained from eq. 23. Subsequently all $A_{n}$ terms can be obtained from eq. 15 . Substituting the $A_{n}$ terms into eq. 13 and eq. 31 yields the analytical solution to the coupled differential equations 5 and 6 subject to the initial/boundary conditions of eq. 7 in the Laplace time domain. Moreover, the cross-sectional average concentration in the Laplace time domain can be calculated from eq. 32.

$$
\phi=-\frac{Z}{\left(D a_{I I}+T\right)} \sum_{n=1}^{\infty} n A_{n}(\lambda)
$$


$c_{m}=2 \sum_{n=0}^{\infty} \frac{A_{n}(\lambda)}{n+2}$

Finally, we need to transform the analytical solution (eq. 13, 31, 32) from the Laplace domain back into the real time domain. Since the Laplace variable $T$ has been absorbed into the formulation and into the roots of the characteristic polynomial given by eq. 21 , we need to perform the Laplace inversion numerically. In this paper, the authors have used the well-known Stehfest (1970) method to perform the inversion. The method is given here by eq. 33 for convenience. In eq. 33, $Y(T)$ is the Laplace transform of $y(t)$, and $N_{L}$ is an even integer. The authors used this method due to its simplicity and thus ease in implementation. Many other Laplace inversion algorithms exist in the literature for which the reader is referred to Cheng (1994) and Duffy (2004). Note that one could either numerically invert the whole solution (i.e. eq. 13, 31 and 32) at once, or only invert $A_{0}$ and use derivative theorems to invert the rest of the $A_{n}$ terms (the former was performed herein). The Laplace inversion is the only numerical step in the solution of the system given by eq. 5-7. This is the reason why the authors refer to it as a semi-analytical solution. It is noteworthy that the steady-state solution of the system given by eq. 5-7 does not require the Laplace transform and our analysis yields a fully-analytical solution to the problem. This is relevant for steady-state Graetz problems in finite or semi-infinite pipes.

$$
\begin{aligned}
& y(t) \square \frac{\ln 2}{t} \sum_{n=1}^{N_{L}} h_{n} Y\left(\frac{n \ln 2}{t}\right) \\
& h_{n}=(-1)^{n+N / 2} \sum_{k=(n+1) / 2}^{\min \left(n, N_{L} / 2\right)} \frac{k^{N / 2}(2 k) !}{(N / 2-k) ! k !(k-1) !(n-k) !(2 k-n) !}
\end{aligned}
$$

We conclude this section with a few remarks. As mentioned earlier, the form of the solution given by eq. 13 was inspired by the Frobenius method for solving ODEs with variable coefficients and regular singular points. In fact, if one were to draw a parallel and form the "indicial equation" for eq. 9 , by insisting $A_{0}(\lambda) \neq 0$ one would obtain a double root of zero for this equation. This together with considering axial symmetry of the concentration field (via eq. 11a) results in the proposed form of the solution (i.e. eq. 13). Therefore, one can imagine how this way of viewing cylindrical problems (among others) both with axial and radial dependence allows for solving a vast number of problems by simply following the proposed steps. This in effect reduces the original variable coefficient partial differential equation into an infinite order ordinary differential equation with constant coefficients. The method allows for the examination of finite pipes (which does not appear to have been pursued by previous investigators) as well as semi-infinite pipes. One might be tempted to take the analogy with the Frobenius method to the extreme and apply it to annular flow geometries. However, non-trivial extensions of the current analysis are required which is outside the scope of this paper. Interestingly enough, the method can also be applied for 2D Cartesian problems (e.g. flow through finite/semi-infinite slits) 
following the same steps outlined above. In this case, eq. 13 would be expanded with respect to the transverse y-coordinate (instead of the radial coordinate) and the resulting analysis would be similar to the power series method in solving ODEs (due to an absence of singularities).

Finally, the formulation of physically meaningful and correct inlet and outlet conditions is a nontrivial task and has been the subject of extensive study (e.g. Liou and Wang, 1990). Among the different choices of inlet/outlet conditions, typically a generalized Dankwerts type boundary condition may be used (taking into account the velocity profile of the fluid within the pipe). This consists of Neumann boundary conditions at the outlet and an inlet condition of the form given by eq. 34 (after non-dimensionalizing and taking the Laplace transform with respect to the time variable). In eq. 34, $W(T)$ represents the Laplace transform of an arbitrary function of time $w(\tau)$.

$-\left.\frac{1}{P e} \frac{\partial \hat{c}}{\partial \lambda}\right|_{\lambda=0^{+}}+\left.\left(1-\xi^{2}\right) \hat{c}\right|_{\lambda=0^{+}}=\left(1-\xi^{2}\right) W(T)$

By comparison of eq. 34 with eq. 11c, it is clear that $a_{2}$ and $F$ are functions of $\xi$. After substituting eq. 13 into eq. 34 and grouping similar $\xi$-power terms we get eq. 35 as the inlet condition for eq. 20a (instead of eq. 24). The analysis then proceeds unaltered from this point forward. Therefore, complex radially dependent inlet/outlet conditions such as eq. 34 can be naturally accommodated into the proposed solution approach. The verification results and examples provided in section 4 , however, focus on relatively simple inlet/outlet conditions.

$$
\left\{\begin{array}{l}
-\frac{1}{P e} A_{0}^{\prime}(0)+A_{0}(0)=W(T) \\
-\frac{1}{P e} A_{2}^{\prime}(0)+A_{2}(0)-A_{0}(0)=-W(T) \\
-\frac{1}{P e} A_{2 m}^{\prime}(0)+A_{2 m}(0)-A_{2 m-2}(0)=0
\end{array} \quad \forall m \geq 2\right.
$$

\section{Results and Discussion}

We consider several special cases and present results obtained from the semi-analytical solution (developed in the previous section). The results are then compared to Computational Fluid Dynamics (CFD) simulations performed in $\mathrm{COMSOL}^{\odot}$, which utilizes the Finite Element method (FEM) to solve eq. 5. In the following, the number of terms used in the series expansion given by eq. 13 was taken in the range $N_{R}=50-74$ and the number of terms used in the numerical inverse Laplace transform (i.e. eq. 33) was taken in the range $N_{L}=12-16$ (where $N_{L}$ is an even integer). The number of elements used in the $\mathrm{COMSOL}^{\odot}$ simulations was 32774 for the semiinfinite ducts, and 12891 for the finite ducts. In both the semi-analytical solution and the 
COMSOL $^{\odot}$ simulations, $N_{R}, N_{L}$ and number of FEM mesh refinements were increased until negligible changes in the results were recorded. In some of the following cases, small but noticeable differences between the two solutions are observed (especially at larger Peclet numbers). The authors ascribe this to the incomplete convergence of the series given by eq. 13 . Complete convergence could not be achieved for these cases, because inclusion of higher order terms in the series (i.e. eq. 13) resulted in numerical instabilities as a result of finite precision arithmetic of computers. For the same reason, the following results could not be pursued to very high Peclet numbers (and are presented for $P e \leq 40$ for semi-infinite ducts and $P e \leq 18$ for finite ducts). This is discussed in further detail in section 4.6, where we present a convergence analysis for two of the presented cases in order to delineate the applicability limit of the solution.

\subsection{Zero Concentration Gradient at the Wall, Semi-Infinite Duct}

A semi-infinite duct with initial conditions given by eq. 7a and boundary conditions given by eq. 36 was considered (with no adsorption and no decay within the fluid bulk):

$\left.c\right|_{\lambda=0}=\left.\left.1 \quad c\right|_{\lambda \rightarrow \infty} \rightarrow 0 \quad \frac{\partial c}{\partial \xi}\right|_{\xi=0,1}=0$

To simulate a semi-infinite duct in $\mathrm{COMSOL}^{\circ}$, a tube of length $L=10 R$ was considered and the simulations were stopped before the outlet boundary was sensed by the evolving concentration field. Figure 1 compares snapshots of radial and axial concentration profiles (at various radial and axial transects) obtained from the semi-analytical solution (shown as solid lines) to those obtained from $\mathrm{COMSOL}^{\odot}$ simulations (shown as circles) at three different Peclet numbers: $P e=0.5,15$ and 40. As can be seen, there is very good agreement between the two and the boundary conditions are clearly satisfied. Figures $1 \mathrm{a}$ and $1 \mathrm{~b}$ correspond to $P e=0.5$ and $\tau=1$, fig. $1 \mathrm{c}$ and $1 \mathrm{~d}$ correspond to $P e=15$ and $\tau=2.25$, and fig. $1 \mathrm{e}$ and $1 \mathrm{f}$ correspond to $P e=40$ and $\tau=6.3$. 


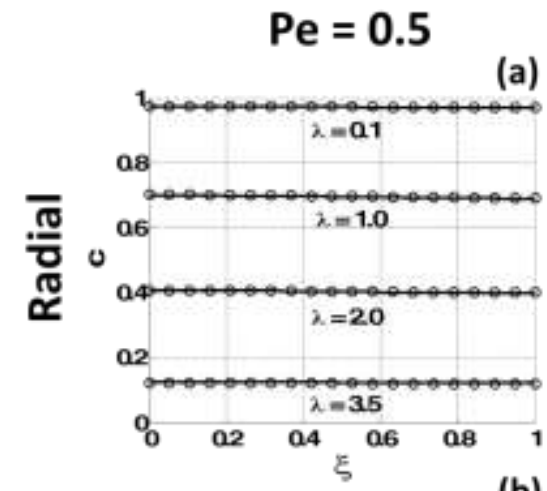

(a)

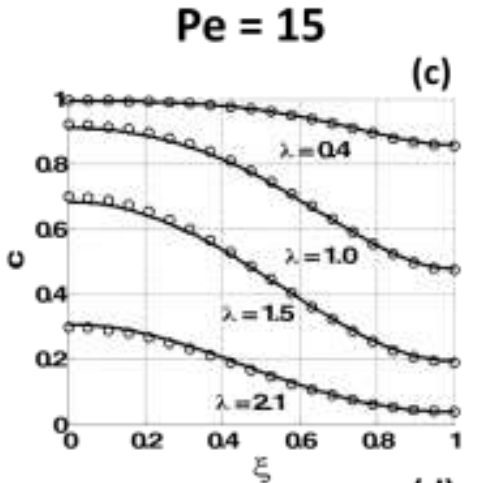

(c)

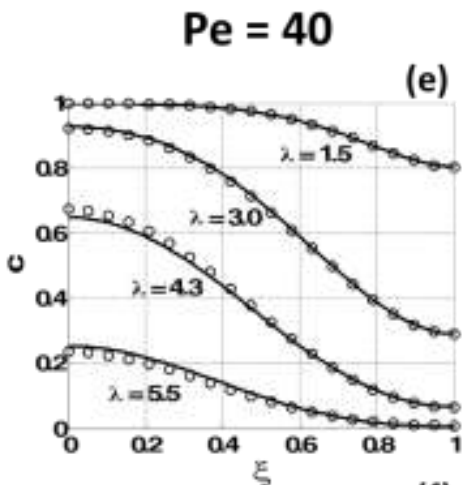

(b)
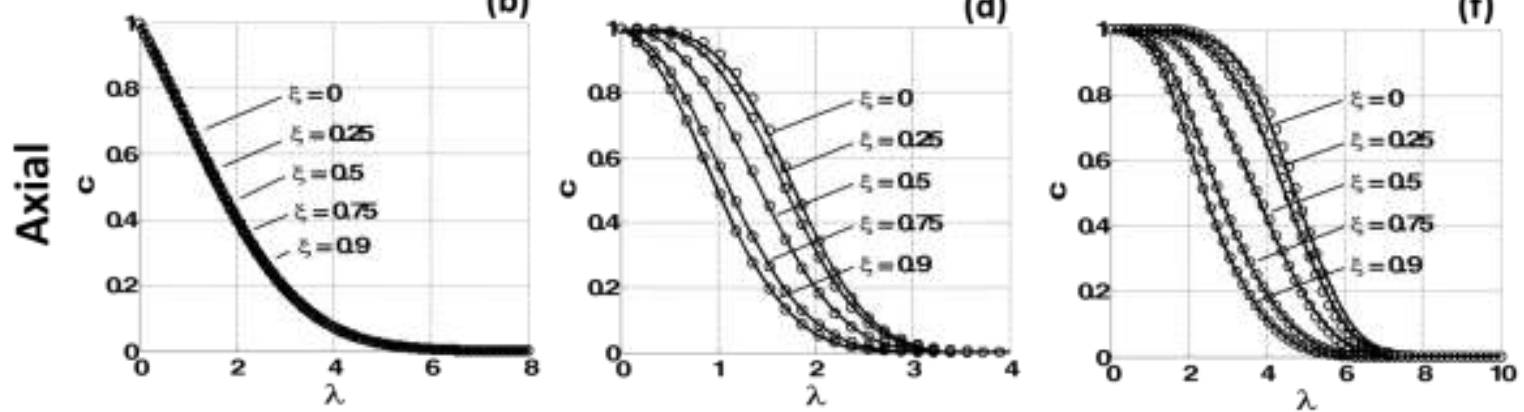

Figure 1: Radial and axial concentration profiles obtained from the semi-analytical solution (solid line) and $\mathrm{COMSOL}^{\odot}$ (circles) at three different Peclet numbers for a semi-infinite tube with zero concentration gradient at the duct wall. (a), (b) correspond to $\tau=1$ and $P e=0.5$, (c), (d) correspond to $\tau=2.25$ and $P e=15$, and (e), (f) correspond to $\tau=6.3$ and $P e=40$.

\subsection{Zero Concentration at the Wall, Semi-Infinite Duct}

The boundary conditions for this case are given by eq. 37 (with no adsorption and no decay within the fluid bulk).

$\left.c\right|_{\lambda=0}=\left.\left.1 \quad c\right|_{\lambda \rightarrow \infty} \rightarrow 0 \quad \frac{\partial c}{\partial \xi}\right|_{\xi=0}=\left.0 \quad c\right|_{\xi=1}=0$

Figure 2 compares the semi-analytical solution to the numerical solution of $\mathrm{COMSOL}^{\odot}$ at three different Peclet numbers: $P e=0.5,15$ and 40. Once again very good agreement between $\mathrm{COMSOL}^{\odot}$ and the semi-analytical solution is observed, which clearly satisfy the boundary conditions imposed. As in the previous section, a tube length of $L=10 R$ was considered to simulate semi-infinite ducts in $\mathrm{COMSOL}^{\odot}$. Figures $2 \mathrm{a}$ and $2 \mathrm{~b}$ correspond to $P e=0.5$ and $\tau=0.25$, fig. $2 \mathrm{c}$ and $2 \mathrm{~d}$ correspond to $P e=15$ and $\tau=7.5$, and fig. $2 \mathrm{e}$ and $2 \mathrm{f}$ correspond to

$P e=40$ and $\tau=7.4$. Note that in this case the concentration field eventually reaches steady state (due to solute outflux at the duct wall), for which the analysis of section 3 provides a fully analytical steady-state solution. 


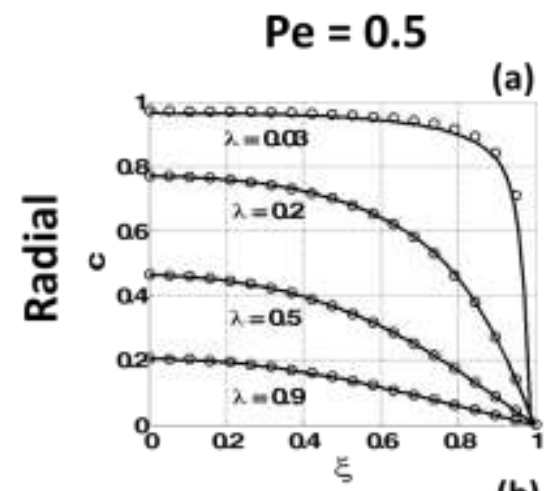

(a)

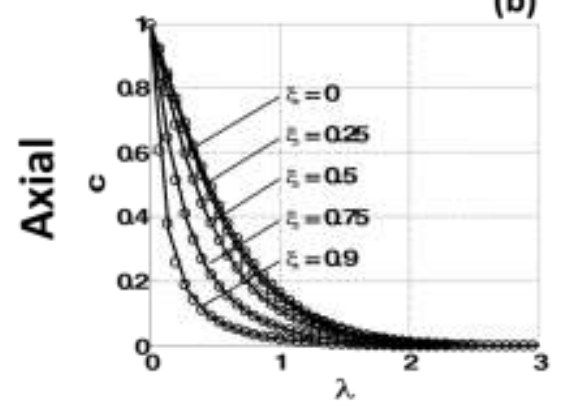

$\mathrm{Pe}=15$
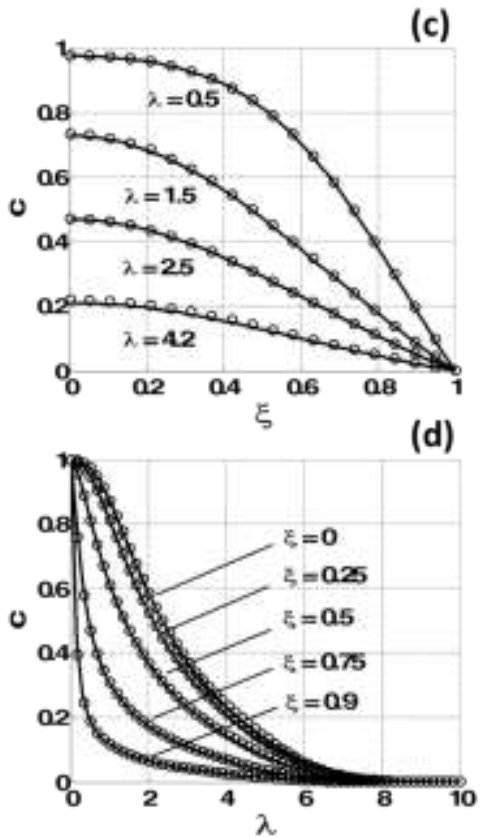

(c)
$\mathrm{Pe}=\mathbf{4 0}$

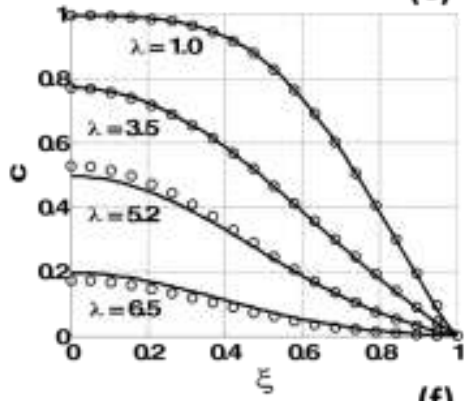

(f)

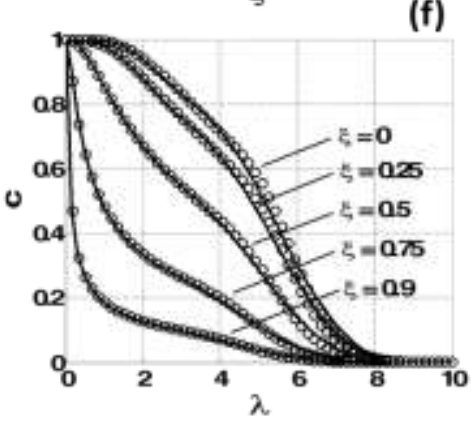

Figure 2: Radial and axial concentration profiles obtained from the semi-analytical solution (solid line) and $\mathrm{COMSOL}^{\odot}$ (circles) at three different Peclet numbers for a semi-infinite tube with zero concentration at the duct wall. (a), (b) correspond to $\tau=0.25$ and $P e=0.5$, (c), (d) correspond to $\tau=7.5$ and $P e=15$, and (e), (f) correspond to $\tau=7.4$ and $P e=40$.

\subsection{Zero Concentration Gradient at the Wall and Outlet, Finite Duct}

The boundary conditions for this case are given by eq. 38 (with no adsorption and no decay within the fluid bulk).

$$
\left.c\right|_{\lambda=0}=\left.1 \quad \frac{\partial c}{\partial \lambda}\right|_{\lambda=1}=\left.0 \quad \frac{\partial c}{\partial \xi}\right|_{\xi=0,1}=0
$$

Figure 3 compares the semi-analytical solution to the numerical solution of $\mathrm{COMSOL}^{\odot}$ at three different Peclet numbers: $P e=0.5,10$ and 18. Since the ducts are finite in length, $\kappa=16$ is chosen ( $\kappa$ defines the aspect ratio of the pipe). Once again very good agreement between $\mathrm{COMSOL}^{\odot}$ and the semi-analytical solution is observed, which satisfy the boundary conditions imposed. The satisfaction of the outlet conditions in figure 3.f may not appear to be very clear. It was verified that this is due to a combination of using finite number of plotting points close to the outlet (in both the CFD and semi-analytical solutions), normalization of the axial coordinate by its length, and suppression of outlet effects due to the slight increase in Peclet number. Figures $3 \mathrm{a}$ and $3 \mathrm{~b}$ correspond to $P e=0.5$ and $\tau=0.32$, fig. $3 \mathrm{c}$ and $3 \mathrm{~d}$ correspond to $P e=10$ and $\tau=1.2$, and fig. $3 \mathrm{e}$ and $3 \mathrm{f}$ correspond to $P e=18$ and $\tau=1.92$. The outlet condition in eq. 38 is 
commonly prescribed for finite length domains and is known as the Danckwerts (1953) exit condition.

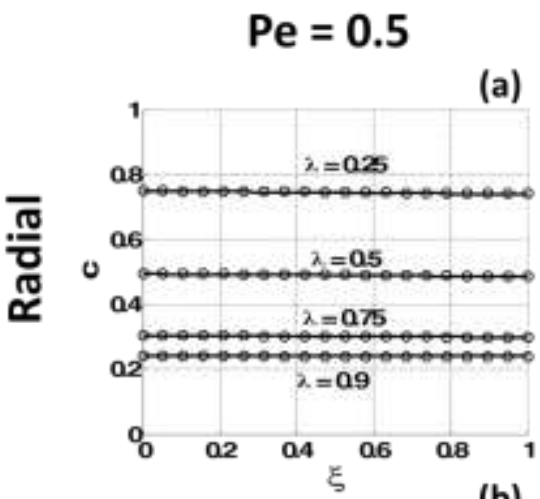

(a)

$\mathrm{Pe}=10$

(c)

$\mathrm{Pe}=18$

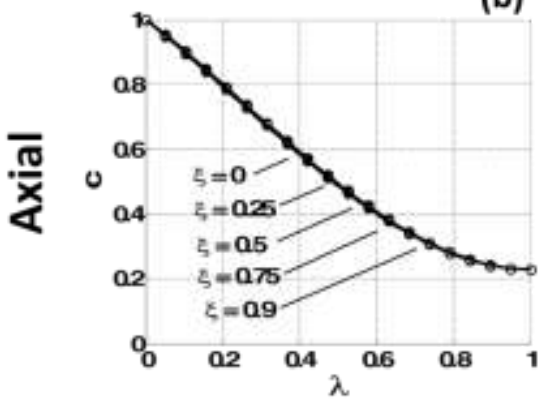

(b)
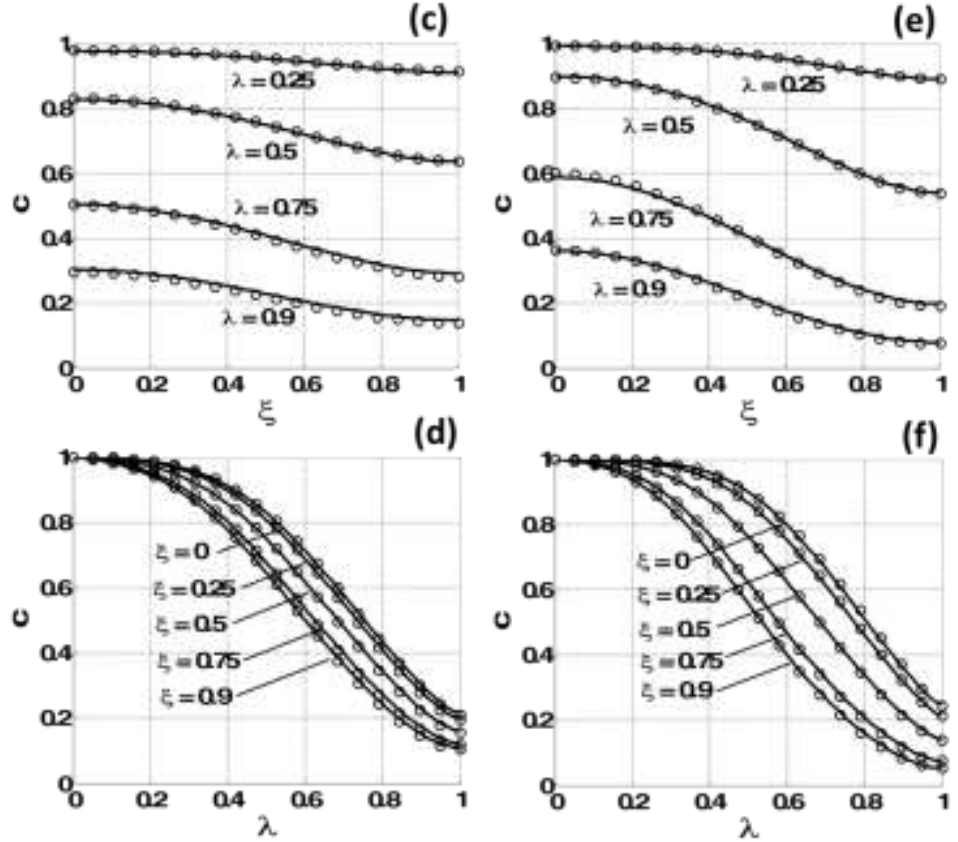

Figure 3: Radial and axial concentration profiles obtained from the semi-analytical solution (solid line) and $\mathrm{COMSOL}^{\odot}$ (circles) at three different Peclet numbers for a finite tube $(\kappa=16)$ with zero concentration gradient at the duct wall and outlet. (a), (b) correspond to $\tau=0.32$ and $P e=0.5$, (c), (d) correspond to $\tau=1.2$ and $P e=10$, and (e), (f) correspond to $\tau=1.92$ and $P e=18$.

\subsection{Zero Concentration Gradient at the Wall and Zero Concentration at the Outlet, Finite Duct}

The boundary conditions for this case are given by eq. 39 (with no adsorption and no decay within the fluid bulk).

$\left.c\right|_{\lambda=0}=\left.1 \quad c\right|_{\lambda=1}=\left.0 \quad \frac{\partial c}{\partial \xi}\right|_{\xi=0,1}=0$

Figure 4 compares the semi-analytical solution to the numerical solution of $\mathrm{COMSOL}^{\odot}$ at three different Peclet numbers: $P e=0.5,10$ and 18. Since the ducts are finite in length, $\kappa=16$ is chosen. Once again very good agreement between $\mathrm{COMSOL}^{\odot}$ and the semi-analytical solution is observed, which clearly satisfy the boundary conditions imposed. Figures $4 \mathrm{a}$ and $4 \mathrm{~b}$ correspond to $P e=0.5$ and $\tau=0.44$, fig. $4 \mathrm{c}$ and $4 \mathrm{~d}$ correspond to $P e=10$ and $\tau=1.25$, and fig. $4 \mathrm{e}$ and $4 \mathrm{f}$ correspond to $P e=18$ and $\tau=1.21$. In fig. 4, notice the formation of a thin boundary layer at the outlet of the duct. Numerical methods typically require substantial refinement in such regions to 
avoid numerical errors, whereas the semi-analytical solution can produce accurate measures of the boundary layer thickness.
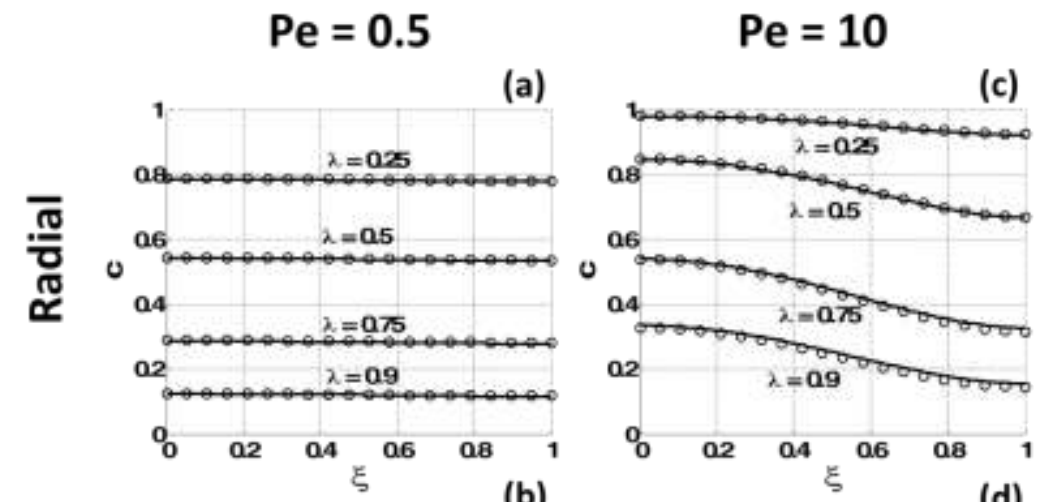

(c)

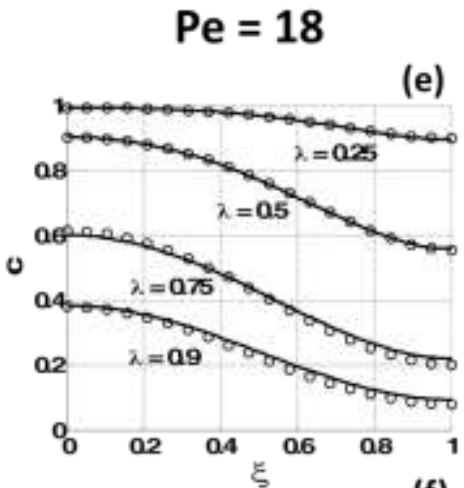

(b)
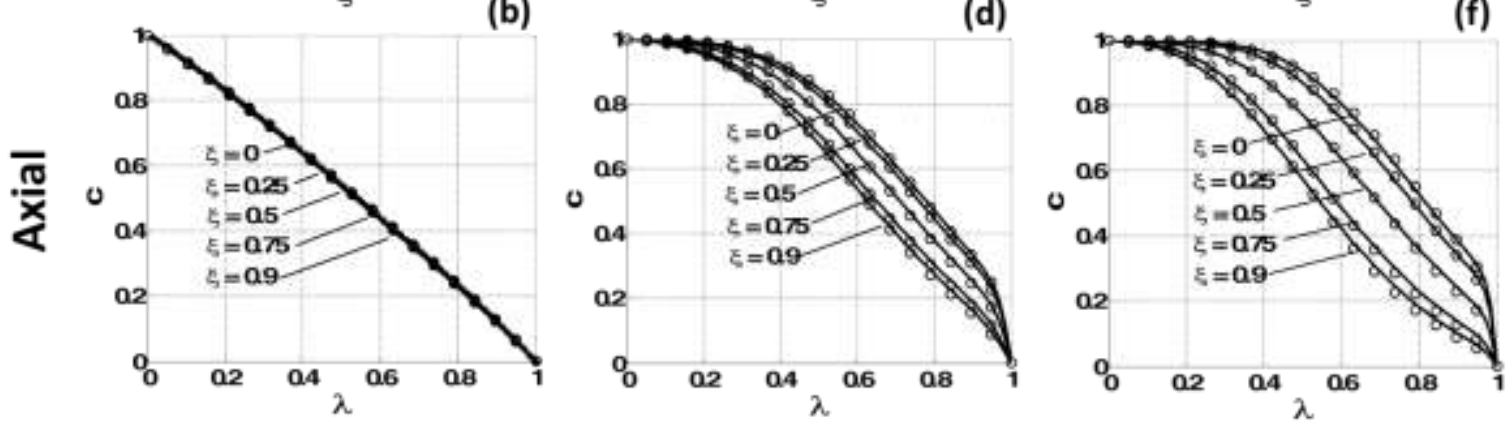

Figure 4: Radial and axial concentration profiles obtained from the semi-analytical solution (solid line) and $\mathrm{COMSOL}^{\odot}$ (circles) at three different Peclet numbers for a finite tube $(\kappa=16)$ with zero concentration gradient at the duct wall and zero concentration at the outlet. (a), (b) correspond to $\tau=0.44$ and $P e=0.5$, (c), (d) correspond to $\tau=1.25$ and $P e=10$, and (e), (f) correspond to $\tau=1.21$ and $P e=18$.

\subsection{Zero Concentration Gradient at the Wall, Semi-Infinite Duct, Power-Law Fluid}

The analysis of section 3 can also be applied to fluids with non-parabolic velocity profiles. This extends further the generality of the solution approach developed to almost arbitrary velocity profiles. As long as the velocity profile is analytic (i.e. has a Taylor expansion) around $r=0$ the analysis remains unchanged (although one may wish to use a truncated version of the Taylor expansion for simplicity). This will naturally result in a different recursive relation for the $A_{n}$ terms than eq. 14 (with the odd terms possibly non-zero). If the velocity profile is not analytic around $r=0$, one can simply fit a low order polynomial to the velocity profile as an approximation (which would loosely be equivalent to the truncated version of its Taylor expansion around a different point).

Here we choose to examine a power-law fluid with a power-law index of $n=1 / 3$ which results in the velocity profile $V(\xi)=V_{0}\left(1-\xi^{4}\right)$. Following the steps of the analysis presented in section 3 , the recursive relation given by eq. 40 is obtained for the $A_{n}$ terms: 


$$
\begin{aligned}
& A_{0}(\lambda) \neq 0 \\
& A_{2}(\lambda)=\frac{\left(T+D a_{I}\right) A_{0}+A_{0}^{\prime}-(1 / P e) A_{0}^{\prime \prime}}{(4 \kappa / P e)} \\
& A_{4}(\lambda)=\frac{\left(T+D a_{I}\right) A_{2}+A_{2}^{\prime}-(1 / P e) A_{2}^{\prime \prime}}{(16 \kappa / P e)} \\
& A_{2 m}(\lambda)=\frac{\left(T+D a_{I}\right) A_{2 m-2}+A_{2 m-2}^{\prime}-A_{2 m-6}^{\prime}-(1 / P e) A_{2 m-2}^{\prime \prime}}{(2 m)^{2}(\kappa / P e)} \\
& A_{2 m-1}(\lambda)=0 \quad \forall m \geq 1
\end{aligned}
$$

Figures $5 \mathrm{a}$ and $5 \mathrm{~b}$ compare radial and axial concentration profiles obtained from the semianalytical solution to those obtained from direct $\mathrm{CFD}$ simulations in $\mathrm{COMSOL}^{\odot}$. Initial and boundary conditions are similar to those in section 4.1 (with no adsorption and no decay within the fluid bulk). These results correspond to $P e=20$ and $\tau=5.57$. A power-law index of $n=1 / 3$ implies shear thinning behavior of the fluid, and thus a relatively flat velocity profile (also higher overall flow rate) compared to the Newtonian counterpart. For the same Peclet number this results in the transport of solute further downstream from the inlet compared to the Newtonian fluid. This is also partly because radial diffusion does not retard the advancing concentration front as much for the power-law fluid, due to low radial concentration gradients (except only near the walls) imposed by the velocity profile. This is clearly seen in figure $5 \mathrm{c}$ for a Newtonian fluid under the same conditions (i.e. maximum fluid velocity, molecular diffusion coefficient and pipe dimensions).

(a)

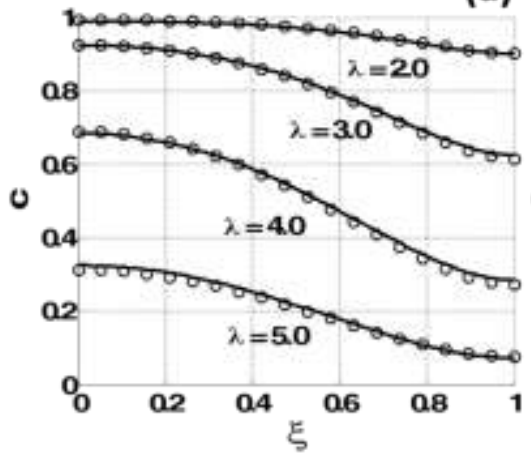

(b)

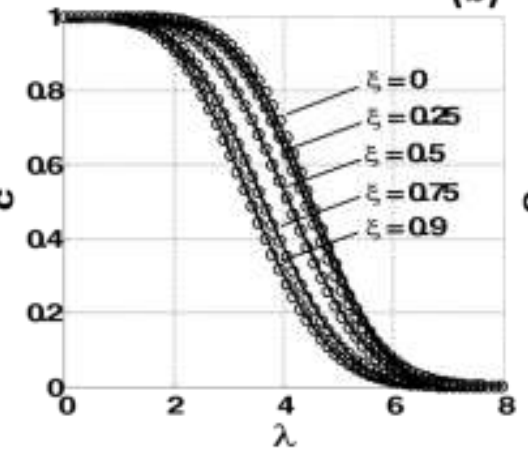

(c)

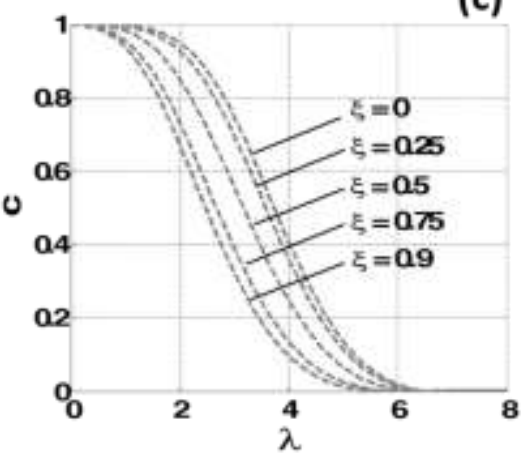

Figure 5: (a) radial and (b) axial concentration profiles obtained from the semi-analytical solution (solid line) and $\mathrm{COMSOL}^{\odot}$ (circles) for a semi-infinite tube and a power-law fluid with a power-law index of $n=1 / 3$, at $P e=20$ and $\tau=5.57$. (c) axial profile of Newtonian fluid under the same conditions.

\subsection{Adsorption and Decay at the Wall, Semi-Infinite Duct}


The boundary conditions for this case are given by eq. 41 (with no decay within the fluid bulk).

$\left.c\right|_{\lambda=0}=\left.\left.1 \quad c\right|_{\lambda \rightarrow \infty} \rightarrow 0 \quad \frac{\partial c}{\partial \xi}\right|_{\xi=0}=\left.0 \quad \frac{\partial c}{\partial \xi}\right|_{\xi=1}=-\operatorname{Sh}\left(\left.c\right|_{\xi=1}-\phi\right)$

Figures $6 \mathrm{a}$ and $6 \mathrm{~b}$ show radial and axial concentration profiles of the bulk fluid at various transects, and figure $6 \mathrm{c}$ shows the deposition profile along the duct wall. These results correspond to $P e=20, S h=10^{6}, D a_{I I}=500, Z=50$, and $\tau=20$, and note that they reflect the boundary condition imposed. Unfortunately these results could not be verified with $\mathrm{COMSOL}^{\odot}$ due to the additional coupled equation 6 (which appears to be not supported by the software). Nevertheless, given the verifications performed in the previous sections, the authors assume that these results bear similar levels of accuracy as the ones already presented.

(a)

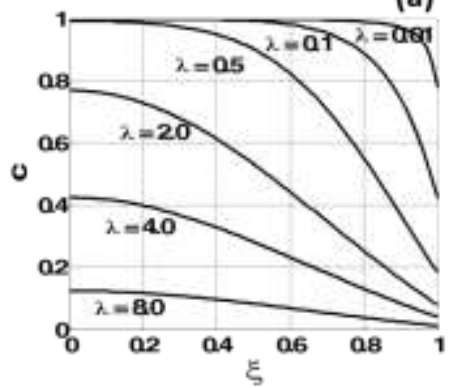

(b)

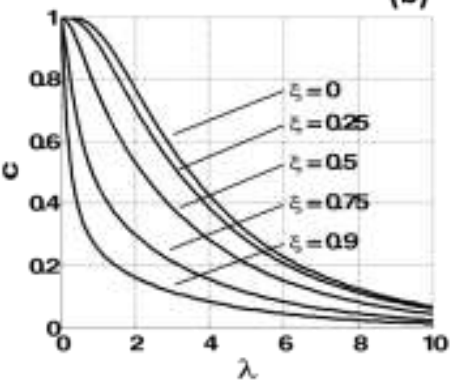

(c)

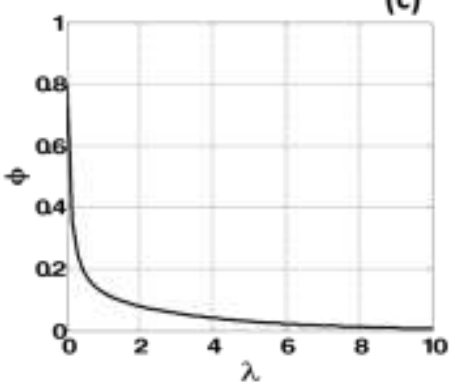

Figure 6: (a) radial and (b) axial fluid bulk concentration profiles obtained from the semi-analytical solution at $\tau=20$ for a semi-infinite tube with adsorption of solute at the duct wall where $P e=20$, $S h=10^{6}, D a_{I I}=500, Z=50$. (c) shows the concentration of the adsorbed solute onto the duct wall at this time.

\subsection{Convergence Analysis}

The rate of convergence of the series given by eq. 13 depends upon the magnitude of the dimensionless parameters that comprise it (e.g. $\mathrm{Pe}$ ). Here, for brevity, we focus on two of the more important cases already discussed, namely the semi-infinite duct of section 4.1 and the finite duct of section 4.3. In both cases, the parameters appearing in the $A_{n}$ terms of eq. 13 consist of $T$ and $P e$, and additionally $\kappa$ for the finite duct case. As $P e$ and $T$ increase the rate of convergence of the series in eq. 13 decreases, requiring more terms for its convergence (i.e. $N_{R}$ needs to increase). However, as $N_{R}$ increases beyond a certain limit the series becomes numerically unstable. The instability is a result of the order of magnitude differences arising within the internal numerics of the solution (mainly due to the recurrence relation given by eq. 14) which leads to the compounding of round-off errors (due to finite precision arithmetic of computers). One instance where this occurs is in the computation of the $\beta_{i}$ coefficients in eq. 23. As $N_{R}$ increases it becomes progressively more difficult to obtain accurate values for the $\beta_{i}$ coefficients, because the system of equations formed by the inlet/outlet conditions, given by eq. 
24 (and eq. 25 for finite ducts), becomes increasingly more ill-conditioned. This poses a computational limit on the largest Peclet number (i.e. $P e$ ) and smallest time (i.e. $\tau$ ) for which we can obtain a meaningfully accurate solution. This should not be very surprising as similar observations of slow convergence rates for early times and high Peclet numbers were made in previously proposed solutions (e.g. Yu, 1976).

The convergence of the series in eq. 13 was studied at various values of $P e, T$ and $\kappa$ for the two cases mentioned. Specifically, for any fixed $P e$ (and $\kappa$ for the finite duct case) the largest value of $T$ (the Laplace time variable) for which eq. 13 converges (without instabilities) within a relative error of $10^{-4}$ was determined. Convergence was considered at four points with $\lambda=0.001,0.01$, $0.1,1$ and $\xi=1$ (where it appeared to be the slowest). These maximum $T$ values were then plotted against $P e$ and a power law function was fitted through the data. Eq. 42 is the inequality that has to be satisfied in order for the series in eq. 13 to converge within a relative error of $10^{-4}$. This inequality, in turn, sets a lower limit for the time variable $\tau$, as they are related via eq. 43 if the Stehfest inversion algorithm (i.e. eq. 33) is used. Combining eq. 42 and 43 we obtain eq. 44 which delineates the smallest time (i.e. $\tau$; as a function of $P e$ and $N_{L}$ ) for which the semianalytical solution can be applied without incurring numerical instabilities. There also exists a maximum $P e$ beyond which the series in eq. 13 becomes numerically unstable regardless of the value of $T$. The $p$ and $q$ fitting parameters as well as the maximum $P e$ are summarized in table 1 for both the semi-infinite and finite duct cases considered. If a different Laplace inversion method is used, only eq. 42 would be of value in deriving an inequality for $\tau$ similar to eq. 44 . Eq. 42 and 44 should be regarded as approximate as the behavior close to the limit may not be very accurate. At early times (where eq. 44 is not satisfied), one may find the solution proposed by Lighthill (1966) complementary to the current solution, since neither the outlet nor the wall boundaries are likely to have been felt by the transporting solute.

$$
\begin{aligned}
T & <p P e^{q} \\
T & =\frac{N_{L} \ln 2}{t} \\
\tau & >\frac{N_{L} \ln 2}{p P e^{q}}
\end{aligned}
$$

Table 1: summary of exponent parameters in eq. 42 and maximum Peclet numbers applicable for the infinite and finite duct cases considered in section 4.6.

\begin{tabular}{lcccccc}
\hline & & Infinite duct & & \multicolumn{3}{c}{ Finite duct } \\
\hline Aspect ratio & $\mathbf{p}$ & $\mathbf{q}$ & $\mathbf{m a x} \mathbf{P e}$ & $\mathbf{p}$ & $\mathbf{q}$ & $\max \mathbf{P e}$ \\
$\boldsymbol{\kappa}=\mathbf{1}$ & 196 & -0.67 & $\sim 60$ & 268 & -0.89 & $\sim 20$ \\
$\boldsymbol{\kappa}=\mathbf{4}$ & - & - & - & 999 & -0.88 & $\sim 40$ \\
$\boldsymbol{\kappa}=\mathbf{1 6}$ & - & - & - & 4507 & -1.0 & $\sim 80$ \\
\hline
\end{tabular}


The authors would like to conclude section 4 with the following comment: among all the previous literature reviewed by the authors, we believe the only method that may have the potential to be applied to finite length ducts is that of Yu's (1976). To that end, the Fourier expansion (instead of the transform) of their infinite system of differential equations (in infinite unknowns) has to be taken, which makes the incorporation of general inlet/outlet conditions such as eq. $7 \mathrm{~d}$ and $7 \mathrm{e}$ very difficult, if not unfeasible. The generality and ease-in-implementation of the current analysis makes it much simpler and thus more favorable to that of Yu's (1976), specifically because instead of dealing with an infinite array of differential equations one only deals with one (i.e. eq. 20a). That said the current analysis has the aforementioned computational limit due to finite precision arithmetic of computers, making it inapplicable to very high Peclet numbers and very early times. For infinite ducts Yu's analysis does not have this issue (although increasingly more terms in their expansion is also required), which seems to be due to the orthogonality of the radial basis chosen (i.e. zero order Bessel functions of first kind, instead of polynomials chosen herein; see eq. 13). Therefore, in order to bridge the gap to higher Peclet numbers, one either has to remove the computational limit of the current semi-analytical solution (through some sort of scaling of the linear system constructed from the inlet/outlet conditions given by eq. 24-25, or perturbation analysis of eq. 20a) or investigate the possibility of extending Yu's work to finite-length ducts, both of which appear to be areas worth exploring further in the future.

\section{Applications}

The problem of solute transport within cylinders is ubiquitous in many engineering and biomedical applications. Therefore the solution approach presented in section 3 may find interest in a number of different fields, a few of which are mentioned in the following.

In nuclear science, high-temperature reactors of fission products are cooled using gaseous Helium. Small leaks of these products into the coolant are transported along cylindrical ducts, which then deposit onto the duct wall and undergo radioactive decay. An accurate estimate of these deposits would allow determining maintenance requirements to minimize any possible health hazards to the operators (Durkee, 1984). Durkee and Lee (1984) provide a solution for the estimation of the deposition profile, but the solution is limited due to the mathematical simplification made (i.e. eq. 45 in their work). Specifically, their approach does not properly solve the transient problem at hand, but simply pre-multiplies the steady-state solution by a moving Heaviside function.

In the chemical and petroleum industry, internal pipeline corrosion is one example where our semi-analytical solution might be utilized. The presence of corrosive constituents such as 
hydrogen sulfide $\left(\mathrm{H}_{2} \mathrm{~S}\right)$, carbon dioxide $\left(\mathrm{CO}_{2}\right)$ and salts within the fluid could cause corrosion of the transporting pipeline. Under appropriate boundary conditions at the pipe wall and neglecting the slight change in pipe diameter due to corrosion, one may calculate the rate of consumption of these solutes at the wall and thus obtain an estimate of axial corrosion profiles. The solution may also find use in the field of microfluidic design and experimentation (see Toepke and Beebe, 2006), where duct diameters are sufficiently small for Peclet numbers to fall within the computational limit of the solution (see section 4.6).

In biomedical/biochemical sciences, intravenous delivery of drugs and bioactive agents into medium/slow flowing blood vessels such as arterioles and capillaries is a scenario in which the axial adsorption or accumulation of concentration near the endothelial cells may pose to be of important interest. Tangelder et al. (1986) studied velocity profiles within mesenteric arterioles of rabbits. A simple calculation shows that the Peclet regime within these arterioles is well within the range of the computational limit of our analysis (see section 4.6). Furthermore, they show that the velocity profile within these arterioles is non-parabolic. In fact, the power-law velocity profile considered in section 4.4 serves as a close approximation. Rugonyi (2008) developed a simplified model for estimating near-wall axial concentration profiles following drug release into blood vessels. Figure $6 \mathrm{c}$ resembles qualitatively the profiles obtained by Rugonyi (2008) (although wall conditions and Peclet regimes differ). Note that the delivery of a finite amount of drug can be modeled by simply changing the inlet boundary condition given by eq. $7 \mathrm{~d}$ to eq. 45 . In eq. $45, \Pi$ is the rectangular function, $c_{0}$ is the amplitude of the pulse injected and $\varepsilon$ determines the duration of the injection period (i.e. $\tau=0$ to $1 / \varepsilon$ ).

$\left.c\right|_{\lambda=0}=c_{0} \Pi(\varepsilon \tau-0.5)$

\section{Conclusions}

We derive a semi-analytical solution to the transient advection-diffusion-reaction equation in finite and semi-infinite ducts. The solution is applicable for general radial- and time-dependent inlet/outlet conditions, complex boundary conditions on the duct wall including adsorption and decay, and arbitrary velocity profiles of the transporting fluid. This is the first time (to the knowledge of the authors) such a solution is proposed for finite length ducts. The only numerical step of the solution is the inverse Laplace transform in the time variable. The approach also produces fully analytical steady-state solutions due to the absence of the Laplace transform step. The solution was verified against computational fluid dynamics (CFD) simulations for various boundary conditions and velocity profiles (Newtonian and power-law), and in all cases good agreement was obtained. In some cases, small but noticeable differences between the CFD and semi-analytical solutions were observed (especially at larger Peclet numbers) which were ascribed to the incomplete convergence of the series given by eq. 13. In these cases, addition of 
higher order terms resulted in numerical instabilities. These instabilities are a result of the order of magnitude differences arising within the internal numerics of the solution which leads to the compounding of round-off errors (due to finite precision arithmetic of computers). Therefore, although theoretically applicable to all regimes, the solution is computationally difficult at very high Peclet numbers and very early times. A convergence analysis was conducted to delineate the boundaries of this limit for two important cases: (a) a semi-infinite duct with Neumann wall conditions, and (b) a finite duct with Neumann wall and outlet conditions. For these cases, the minimum time and maximum Peclet number for which reliable results, devoid of said instabilities, could be obtained from the semi-analytical solution can be determined from eq. 44 and table 1. At early times (where eq. 44 is not satisfied), one may find the solution proposed by Lighthill (1966) complementary to the current solution, since neither the outlet nor the wall boundaries are likely to have been felt by the transporting solute.

The derivation of the solution involved using a new approach for solving two-dimensional partial differential equations (PDEs) with non-constant coefficients, which was inspired by the Frobenius (and power series) method for solving ordinary differential equations (ODEs). The approach converts the original variable coefficient PDE to a single infinite-order ODE with constant coefficients. Although applied here to problems in cylindrical coordinates, the method is applicable to Cartesian problems (e.g. flow through slits) as well. Thus, the method is suspected to provide solutions for a large class of PDEs of this type. The current solution may find

applications in a number of engineering and/or biomedical fields, it can be used to verify numerical simulators and serve as a simple and easy-to-implement alternative where access to numerical simulators is not available.

\section{Acknowledgements}

This material is based upon work supported as part of the Center for Frontiers of Subsurface Energy Security, an Energy Frontier Research Center funded by the U.S. Department of Energy, Office of Science, and Office of Basic Energy Sciences under Award Number DE-SC0001114.

\section{References}

Ananthakrishnan, V., W. N. Gill, and A. J. Barduhn. "Laminar dispersion in capillaries: Part I. Mathematical analysis." AIChE Journal 11, no. 6 (1965): 1063-1072.

Aris, R. "On the dispersion of a solute in a fluid flowing through a tube." Proceedings of the Royal Society of London. Series A. Mathematical and Physical Sciences 235, no. 1200 (1956): 67-77. 
Barron, R. F., X. Wang, T. A. Ameel, and R. O. Warrington. "The Graetz problem extended to slip-flow." International Journal of Heat and Mass Transfer 40, no. 8 (1997): 1817-1823.

Cheng, A. HD, P. Sidauruk, and Y. Abousleiman. "Approximate inversion of the Laplace transform." Mathematica Journal 4, no. 2 (1994): 76-82.

Chun, D. H. "Distribution of concentration in flow through a circular pipe." International Journal of Heat and Mass Transfer 13, no. 4 (1970): 717-723.

Danckwerts, P. V. "Continuous flow systems: distribution of residence times." Chemical Engineering Science 2, no. 1 (1953): 1-13.

Duffy, D. G. Transform methods for solving partial differential equations. CRC press, 2004.

Durkee Jr, J. W., and C. E. Lee. "An analytic benchmark solution to the linear, time-dependent isothermal laminar flow fission-product plateout problem." Annals of Nuclear Energy 11, no. 7 (1984): 337-345.

Durst, F., S. Ray, B. Ünsal, and O. A. Bayoumi. "The development lengths of laminar pipe and channel flows." Journal of fluids engineering 127, no. 6 (2005): 1154-1160.

Gill, W. N., and R. Sankarasubramanian. "Exact analysis of unsteady convective diffusion." Proceedings of the Royal Society of London. A. Mathematical and Physical Sciences 316, no. 1526 (1970): 341-350.

Gill, W. N., and R. Sankarasubramanian. Discussion: "On Laminar Dispersion for Flow Through Round Tubes" (Yu, J. S., 1979, ASME J. Appl. Mech., 46, pp. 750-756). J. Appl. Mech. 47(4), 975-976 (1980), doi: 10.1115/1.3153832.

Graetz, L., Über die Wärmeleitungsfähigkeit von Flüssigkeiten, part 1. Annalen der Physik und Chemie, 1883, 18, 79-94; part 2, 1885, 25, 337-357.

Hunt, B. "Diffusion in laminar pipe flow." International Journal of Heat and Mass Transfer 20, no. 4 (1977): 393-401.

Jeong, HE, and JT Jeong. "Extended Graetz problem including axial conduction and viscous dissipation in microtube." Journal of mechanical science and technology 20, no. 1 (2006): 158166.

Lahjomri, J., and A. Oubarra. "Analytical solution of the Graetz problem with axial conduction." Journal of heat transfer 121, no. 4 (1999): 1078-1083. 
Lighthill, M. J. "Initial development of diffusion in Poiseuille flow." IMA Journal of Applied Mathematics 2, no. 1 (1966): 97-108.

Liou, CT, and FS Wang. "Solutions to the extended Graetz problem for a power-model fluid with viscous dissipation and different entrance boundary conditions." Numerical heat transfer 17, no. 1 (1990): 91-108.

Rugonyi, S. "Effect of blood flow on near-the-wall mass transport of drugs and other bioactive agents: A simple formula to estimate boundary layer concentrations." Journal of biomechanical engineering 130, no. 2 (2008): 021010.

Shankar, A., and A. M. Lenhoff. "Dispersion in laminar flow in short tubes." AIChE journal 35, no. 12 (1989): 2048-2052.

Stehfest, H. "Algorithm 368: Numerical inversion of Laplace transforms [D5]." Communications of the ACM 13, no. 1 (1970): 47-49.

Tangelder, G. J., D. W. Slaaf, A. M. Muijtjens, T. Arts, M. G. Oude Egbrink, and R. S. Reneman. "Velocity profiles of blood platelets and red blood cells flowing in arterioles of the rabbit mesentery." Circulation Research 59, no. 5 (1986): 505-514.

Taylor, G. "Dispersion of soluble matter in solvent flowing slowly through a tube." Proceedings of the Royal Society of London. Series A. Mathematical and Physical Sciences 219, no. 1137 (1953): 186-203.

Toepke, M. W., and D. J. Beebe. "PDMS absorption of small molecules and consequences in microfluidic applications." Lab Chip 6, no. 12 (2006): 1484-1486.

Tseng, C. M., and R. W. Besant. "Transient heat and mass transfer in fully developed laminar tube flows." International Journal of Heat and Mass Transfer 15, no. 2 (1972): 203-215.

Yu, J. S. "An approximate analysis of laminar dispersion in circular tubes." Journal of Applied Mechanics 43, no. 4 (1976): 537-542.

Yu, J. S. "On laminar dispersion for flow through round tubes." Journal of Applied Mechanics 46, no. 4 (1979): 750-756.

Yu, J. S. "Dispersion in laminar flow through tubes by simultaneous diffusion and convection." Journal of Applied Mechanics 48, no. 2 (1981): 217-223. 